\title{
Croissance et assimilation nette foliaire de jeunes plants de dix arbres de la forêt guyanaise, cultivés à cinq niveaux d'éclairement
}

\author{
Têtè Sévérien Barigahª ${ }^{\mathrm{a}}$ Pascal Imberta, Roland Huc ${ }^{\mathrm{b}}$ \\ aStation de recherches forestières, Inra B.P. 709, 97387 Kourou cedex, France \\ bLaboratoire de recherches forestières méditerranéennes, \\ Inra Avenue A. Vivaldi 84000 Avignon, France
}

(Reçu le 29 juillet 1996 ; révisé le 19 décembre 1996 ; accepté le 22 décembre 1997)

\begin{abstract}
Growth and net assimilation rate of seedlings of ten Guianan tree species grown under five light regimes. Seedlings of ten species of tropical rainforest trees (Bocoa prouacensis, Carapa guianensis, Carapa procera, Jacaranda copaia, Platonia insignis, Qualea rosea, Recordoxylon speciosum, Sterculia excelsa, Virola surinamensis, Vouacapoua americana) occurring in successional phases were grown in shade tunnels under five relative light levels $(65 \%, 45 \%, 25 \%, 10 \%$ and $4 \%$ of full sunshine). The study was designed to assess the behaviour of the species in juvenile stages versus different light growth conditions. Mainly net assimilation and growth rates were recorded. For seedlings growing under $65 \%$ of full sunlight, maximum net assimilation rates were fairly variable between the species and ranged from 3.0 to 7 $\mu \mathrm{mol} \mathrm{m} \mathrm{m}^{-2} \mathrm{~s}^{-1}$ and the total dry matter production ranged from 8 to $286 \mathrm{~g}$ per plant for plants grown for 20 months. The net assimilation rate of Carapa guianensis decreased by almost $40 \%$ between $45 \%$ and $65 \%$ of full sunlight. The growth performance was substantially reduced at the lowest irradiance, but the pattern varied highly between species with increasing light intensity. The light level for optimal growth for most species ranged between $25 \%$ and $45 \%$ of full sunlight. Nevertheless, Bocoa prouacensis, a late successional species, had a higher growth performance under the highest light intensity tested $(65 \%)$ than in lower light environments $(<45 \%)$.(@ Inra/Elsevier, Paris.)
\end{abstract}

\section{tropical rainforest / net assimilation rate / growth rate / stem volume index}

Résumé - Des plantules de dix espèces d'arbres tropicaux (Bocoa prouacensis, Carapa guianensis, Carapa procera, Jacaranda copaia, Platonia insignis, Qualea rosea, Recordoxylon speciosum, Sterculia excelsa, Virola surinamensis, Vouacapoua americana) ont été élevées dans des serres tunnels sous différents régimes lumineux $(65 \%, 45 \%, 25 \%, 10 \%$ et $4 \%$ du plein découvert). L'objectif est de préciser le comportement des espèces au stade juvénile vis-à-vis de différentes conditions d'éclairement. L'assimilation nette foliaire et la croissance des plantules de ces espèces ont été plus particulièrement étudiées. Les valeurs maximales d'assimilation nette foliaire mesurées sur les plantules soumises à $65 \%$ du plein éclairement étaient différentes d'une espèce à l'autre et étaient comprises entre 3,0 et $7,2 \mu \mathrm{mol} \mathrm{m}^{-2} \mathrm{~s}^{-1}$ tandis que la matière sèche 
totale produite par ces plantes âgées de vingt mois était comprise entre 8 et $286 \mathrm{~g}$ par plante. L'assimilation nette foliaire a été plus faible dans les conditions de faible éclairement. Chez l'espèce Carapa guianensis, une réduction d'environ $40 \%$ du taux d'assimilation nette foliaire a été observée pour des niveaux d'éclairement compris entre $45 \%$ et $65 \%$ du plein découvert. La croissance maximale a sensiblement diminuée dans les conditions de faible éclairement et les taux de croissance étaient différents d'une espèce à l'autre lorsque les plantules étaient cultivées sous un régime lumineux plus fort. L'optimum de croissance, dans les conditions d'expérience, était compris entre $25 \%$ et $45 \%$ du plein découvert. Cependant, Bocoa prouacensis, espèce des stades tardifs de la succession, a présenté une croissance plus élevée pour l'intensité lumineuse la plus forte $(65 \%)$ que pour de plus faibles valeurs d'éclairement $(<45 \%)$. (C) Inra/Elsevier, Paris.)

forêt tropicale humide / assimilation nette foliaire / vitesse de croissance / indice de volume

\section{INTRODUCTION}

La forêt tropicale humide est un écosystème très complexe, caractérisé par des sols souvent acides et pauvres en éléments minéraux et en humus et par une très grande diversité d'espèces arborées. Chacune de ces espèces se caractérise par un comportement différent vis-à-vis de la lumière, de la température, de l'eau et des substances nutritives à différents stades de leur vie $[40,51]$.

Le rôle moteur des perturbations (chute de branche, volis, chablis...) sur la dynamique des régénérations naturelles et sur la croissance des juvéniles a été démontré [15]. Ces perturbations sont de nature à générer des variations de lumière en quantité et en qualité, des variations de température ainsi que d'humidité de l'air et du sol. Ainsi, dans le sous-bois des forêts tropicales humides non perturbées, à peine $1 \%$ du rayonnement solaire parvient au sol à travers la voûte forestière [7] conditionnant à la fois l'assimilation carbonée, la demande évaporative et la survie des plantules. La survie d'une plante, dans des conditions environnementales données, dépend essentiellement de sa capacité à résister à l'épuisement de ses réserves en maintenant un bilan carboné positif ou nul [21].

Les connaissances relatives au fonctionnement des essences des forêts tropi- cales humides sont encore éparses [6, 33] et les conséquences à long terme de l'exploitation forestière sont peu connues (que ce soit sur le plan de la dynamique induite, de la qualité des bois restant sur pied et destinés à former les futures grumes ou plus globalement de la régénération). C'est pourquoi, il est important de connaître les besoins en lumière des jeunes plants (base de la régénération) des principales essences et leur sensibilité (évaluée en terme de croissance) à l'énergie lumineuse disponible dans le sousbois.

Ce travail a pour objet d'étudier, en conditions semi-contrôlées, la réponse à l'éclairement de l'assimilation nette foliaire et de la croissance de jeunes plants de dix espèces d'arbres tropicaux. Cette étude devrait permettre à terme de connaître les besoins en énergie lumineuse des principales espèces de la forêt tropicale de Guyane et d'établir des groupes fonctionnels d'espèces en vue de leur utilisation en sylviculture.

Des plantules de quelques espèces ayant un intérêt commercial et/ou présentant des caractéristiques écologiques a priori différentes $[8,16]$ ont été cultivées en condition de serre-tunnel et sous différents régimes lumineux. L'assimilation nette foliaire, la croissance en hauteur et en biomasse de jeunes plants de ces espèces ont été étudiées car c'est sur les 
jeunes plants (plus fragiles, moins bien enracinés) que s'exercent en premier les contraintes climatiques majeures.

\section{MATÉRIEL ET MÉTHODES}

\subsection{Matériel végétal}

Dix espèces d'arbres de la forêt tropicale de Guyane (Bocoa prouacensis, Carapa guianensis, Carapa procera, Jacaranda copaia, Platonia insignis, Qualea rosea, Recordoxylon speciosum, Sterculia excelsa, Virola surinamensis, Vouacapoua americana) ont été choisies parmi celles retenues par le programme Régénération Naturelle de l'Inra et celui du Cirad-Forêt $[5,16,19]$ en fonction de leur vitesse d'apparition après une perturbation naturelle ou d'origine anthropique de la forêt primaire. Ces espèces (annexe 1) ont été réparties dans différents sous-groupes (tableau I) : intolérants (pionniers), semi-tolérants (opportunistes ou encore colonisatrices des trouées) et tolérants (tardifs de la succession) en fonction de la capacité des plants dans les jeunes stades à supporter l'ombrage $[1,30,40]$ et de la place des arbres dans la structure verticale au stade adulte [52]. Riddoch et al. [44] ont déjà souligné les insuffisances de ce mode de répartition des espèces du fait de l'existence de nombreuses espèces charnières entre deux groupes écologiques contigus de la succession.

Les plantules utilisées ont été obtenues à partir de graines à l'exception de celles de Jacaranda copaia dont la taille des graines et leur mode de dispersion ont conduit à recourir au repiquage des semis (stade 2 à 3 feuilles) prélevés à la lisière de la forêt et/ou dans de grandes trouées. Dix blocs composés chacun d'un individu par espèce ont été installés dans chaque serre de manière à éliminer la compétition entre les plants pour les ressources du milieu. Néanmoins, une compétition (non quantifiée) pour la lumière, consécutive au développement des plantes à croissance rapide, a apporté un ombrage supplémentaire aux plants qui leur étaient contigus. La position d'une espèce à l'intérieur d'un bloc a été déterminée de façon aléatoire pour le premier bloc mis en place. Cette disposition initiale a été répétée lors de la constitution des autres blocs.

\section{Environnement climatique}

Le climat du site expérimental de Paracou $\left(5^{\circ} 15^{\prime} \mathrm{N} ; 52^{\circ} 55^{\prime} \mathrm{O}\right)$ est de type équatorial [10]. Il est caractérisé par des pluies abondantes, plus de $3000 \mathrm{~mm}$ par an avec deux saisons des pluies de novembre à février et d'avril à juillet. L'humidité relative moyenne de l'air est importante et peu variable ( 80 à $90 \%$ ). La température est presque constante dans l'année (environ $26^{\circ} \mathrm{C}$ ), mais on note des écarts diurnes nets, de 6 à $12{ }^{\circ} \mathrm{C}$ suivant les saisons.

Les expérimentations ont été conduites dans cinq serres-tunnels $(\mathrm{L}=10,50 \mathrm{~m}$; $l=6,5 \mathrm{~m}$ et $\mathrm{h}=2,5 \mathrm{~m}$ ) recouvertes (sauf aux extrémités) d'une ou de plusieurs couches d'ombrière permettant d'atténuer le rayonnement solaire (plein découvert) et de créer ainsi, dans les serres, différents niveaux d'éclairement (éclairement relatif) susceptibles d'être rencontrés en forêt.

Les conditions générales de croissance (microclimat dans les serres) sont les suivantes.

- La lumière, un éclairement relatif moyen (calculé à partir du rapport de l'intensité lumineuse mesurée, par temps clair, à l'intérieur de la serre à celle mesurée en plein découvert) qui a été de $65 \%$ ( $\left.1400 \mu \mathrm{mol} \mathrm{m}^{-2} \mathrm{~s}^{-1}\right), 45 \%$ (1 $\left.000 \mu \mathrm{mol} \mathrm{m} \mathrm{m}^{-2} \mathrm{~s}^{-1}\right), 25 \%\left(500 \mu \mathrm{mol} \mathrm{m} \mathrm{m}^{-2}\right.$ $\left.\mathrm{s}^{-1}\right), 10 \%\left(200 \mu \mathrm{mol} \mathrm{m} \mathrm{m}^{-2} \mathrm{~s}^{-1}\right)$ et $4 \%(90$ $\mu \mathrm{mol} \mathrm{m} \mathrm{m}^{-2} \mathrm{~s}^{-1}$ ). Le plan d'expérience n'a pas prévu d'essai en plein éclairement (100\%) à cause de la sensibilité de la plupart des essences locales, dans leurs stades jeunes, aux conditions du plein découvert $[1,11]$.

- La température, celle de l'air ambiant modifiée par la présence des ombrières [1, 46]. Entre 12 et $16 \mathrm{~h}$, l'écart thermique entre la serre à fort éclairement et la serre à faible éclairement était de 2 à $3{ }^{\circ} \mathrm{C}$. La température était comprise entre 27 et $38{ }^{\circ} \mathrm{C}$ consécutive aux variations thermiques diurnes saisonnières.

- L'humidité, comprise entre 80 et $95 \%$.

- L'alimentation hydrique, un régime non limitant qui a consisté en un apport quotidien 
de 0,20 à $0,35 \mathrm{~L}$ d'eau par plant au moyen d'un dispositif automatique d'arrosage par goutte à goutte en fin de nuit.

- Le sol, de la terre végétale (horizon A d'un sol de forêt primaire) à laquelle il na pas été rajouté d'engrais a servi à remplir des pots plastiques de $11 \mathrm{~L}$.

\subsection{Méthodes}

Les mesures effectuées ont concerné essentiellement les échanges gazeux, la croissance en hauteur et diamètre et les biomasses aérienne et racinaire.

Des mesures périodiques d'assimilation nette foliaire ont été effectuées de juillet à décembre 1992 entre 10 et $13 \mathrm{~h}$ à raison d'une ou deux campagnes hebdomadaires à l'aide d'un analyseur de $\mathrm{CO}_{2}$ à infrarouge Li-6200 (Li-cor, Lincoln, Nebraska). Les mesures d'assimilation nette foliaire ont été effectuées dans les conditions de culture des plants (la source de lumière était le rayonnement solaire maximal disponible dans chacune des serres). Les conditions météorologiques ont été déterminantes dans le choix des créneaux horaires pour la collecte de données. Avant 10 h, le soleil n'est pas suffisamment haut dans le ciel pour que l'intensité lumineuse soit maximale dans les serres. Au-delà de 13 h, la température devient trop importante par rapport au début de la matinée (l'amplitude thermique peut atteindre $12{ }^{\circ} \mathrm{C}$ ). Pour que les mesures soient effectuées approximativement dans les mêmes conditions de lumière, aucune prise de données n'a été effectuée tant que des nuages pouvaient occulter les rayons du soleil. En moyenne, la collecte de données a porté sur deux feuilles par plant à raison de quatre à cinq plants par espèce, par serre et par campagne de mesures. Les mesures d'assimilation nette foliaire ont toujours été effectuées sur des feuilles adultes non sénescentes (feuilles appartenant au second verticille ou bien sur des feuilles de rang $3 \mathrm{et} / \mathrm{ou} 4$ à partir de du bourgeon terminal de la tige pour tenir compte de l'effet de l'âge sur les performances de l'appareil photosynthétique foliaire [3, 13].

Des mesures non destructives de hauteur (h) et de diamètre (d) à $22 \mathrm{~cm}$ du sol ont été effectuées tous les 2 mois pendant 23 mois (jusqu'au moment où les bourgeons terminaux des plus grandes plantes ont commencé à toucher le toit de la serre) à partir du troisième mois après la plantation. Tous les plants vivants ont alors été récoltés. La surface foliaire, la hauteur et le diamètre à $22 \mathrm{~cm}$ du sol ont été mesurés pour chaque plant ; les différents organes (limbe, pétiole, tige, rameau, pivot et chevelu racinaire) ont été séparés puis mis à sécher à l'étuve à $80^{\circ} \mathrm{C}$ pendant $10 \mathrm{j}$. La biomasse produite par espèce et par organe a été estimée à partir de la pesée de la matière sèche obtenue (somme du poids sec du limbe, du pétiole, de la tige et des rameaux pour la biomasse aérienne et somme du poids sec du pivot et du chevelu racinaire pour la biomasse souterraine; la biomasse totale étant la somme des biomasses aérienne et souterraine). Le produit hauteur par diamètre au carré $\left(h d^{2}\right)$, appelé indice de volume a été utilisé pour estimer le volume de la tige des plantules étudiées dans ce travail.

\section{RÉSULTATS ET DISCUSSION}

\subsection{Assimilation nette foliaire}

Les graphiques de la figure 1 représentent l'assimilation nette foliaire des différentes espèces en fonction de l'intensité lumineuse maximale dans les serres. La réponse des feuilles à la lumière montre que l'assimilation nette foliaire diffère d'une espèce à l'autre. Cependant, du fait de la similitude de leur comportement, ces espèces peuvent être classées en trois groupes :

- les espèces comme Jacaranda copaia, Qualea rosea, Recordoxylon speciosum, Sterculia excelsa et Virola surinamensis qui présentent un plateau de saturation net pour des faibles valeurs de rayonnement (inférieures ou égales à $45 \%$ du plein découvert);

- les espèces comme Bocoa prouacensis, Carapa procera, Platonia insignis et Vouacapoua americana chez lesquelles le plateau de saturation se situerait audelà de $45 \%$ du plein découvert ; 
- les espèces comme Carapa guianensis pour lesquelles l'assimilation nette foliaire baisse sensiblement lorsque l'intensité lumineuse dépasse un certain seuil.

On peut noter qu'à fort éclairement (65\% du plein découvert), les valeurs les plus élevées d'assimilation foliaire nette $\left(7,2 \mu \mathrm{mol} \mathrm{m} \mathrm{m}^{-2} \mathrm{~s}^{-1}\right)$ ont été mesurées sur l'espèce pionnière $J$. copaia tandis que les plus faibles $\left(3,0 \mu \mathrm{mol} \mathrm{m} \mathrm{m}^{-2} \mathrm{~s}^{-1}\right)$ l'ont été sur l'espèce opportuniste Qualea rosea. L'assimilation nette maximale varie d'un facteur 1 à 2,4 entre les espèces. À faible éclairement (4\% du plein découvert), les valeurs les plus élevées d'assimilation foliaire nette $(1,5$ $\mu \mathrm{mol} \mathrm{m}{ }^{-2} \mathrm{~s}^{-1}$ ) ont été enregistrées sur les plantules de Qualea rosea et les plus faibles valeurs d'assimilation nette foliaire $\left(0,5 \mu \mathrm{mol} \mathrm{m} \mathrm{m}^{-2} \mathrm{~s}^{-1}\right)$ sur une espèce d'ombre Recordoxylon speciosum. La gamme de variation dans ce cas est d'un facteur 1 à 3 entre les espèces. Il y a donc une grande variabilité du taux d'assimilation nette maximale entre les espèces étudiées comme c'est le cas chez de nombreux arbres [14, 31]. Cette variabilité pourrait s'expliquer par des différences de caractéristiques foliaires telles que la masse surfacique, les conductances stomatique et mésophyllienne $[18,38]$. De nombreux travaux $[33,42,44,50]$ ont montré le rôle important de ces caractéristiques dans le contrôle de la croissance des plantes lorsqu'elles sont soumises à différents niveaux d'éclairement. Ces différents aspects du fonctionnement des espèces n'ont pas été abordés dans ce travail.

Certains auteurs $[6,32,48]$ en s'appuyant sur des connaissances empiriques, ont réalisé un classement des espèces en fonction de leur capacité photosynthétique mais les données disponibles dans ce travail montrent à l'inverse que le taux d'assimilation nette foliaire ne constitue pas un critère suffisant pour présumer, a priori, du statut d'apparition (pionniers, opportunistes et les tardifs de la succession) des espèces au cours de la succession forestière. Nos résultats par ailleurs confirment les conclusions issues des travaux effectués sur d'autres plantules d'espèces tropicales : Milicia excelsa et Blighia sapida [44], Amanoa caribea, Dacryodes excelsa, Simaruba amara, Symphonia globulifera, Richeria grandis, Byrsonima coriacea, Podocarpus coriaceus [17].

Les valeurs maximales d'assimilation nette foliaire mesurées dans nos conditions (l'éclairement maximal étant de $1400 \mu \mathrm{mol} \mathrm{m} \mathrm{m}^{-2} \mathrm{~s}^{-1}$ ) sont faibles mais restent comparables à celles de nombreux auteurs [17, 44, 45, 48].

\subsubsection{Mortalité}

Le nombre de plants morts (compris entre 0 et 3) sur un total de dix plants par espèce et par serre (régime lumineux) est faible. La comparaison des effectifs initiaux et finaux, en utilisant le test du $\chi^{2}$ n'a pas permis de déceler des différences significatives de mortalité entre les plantes de même espèce soumises à différents régimes lumineux. On ne peut donc pas conclure quant à l'influence des conditions de croissance sur la survie des plants.

Le statut de pionnier laisse a priori supposer qu'au stade jeune, les plantules ont besoin de beaucoup de lumière pour survivre et se développer [52] ; on peut donc s'attendre à ce que le taux de mortalité de ces plantes soit élevé en condition d'ombrage ou de semi-ombrage et qu'à l'inverse, les plantes d'ombre meurent davantage dans des conditions de forts éclairements comme chez les plantules de certaines Dipterocarpaceae $[37,46]$. Les données, relatives à la mortalité des plants dans les différentes conditions de croissance, recueillies dans ce travail ne sont donc pas en accord avec cette hypothèse. Il n'a pas non plus été observé des vagues périodiques de mortalité des plantules comme le laissait prévoir des 

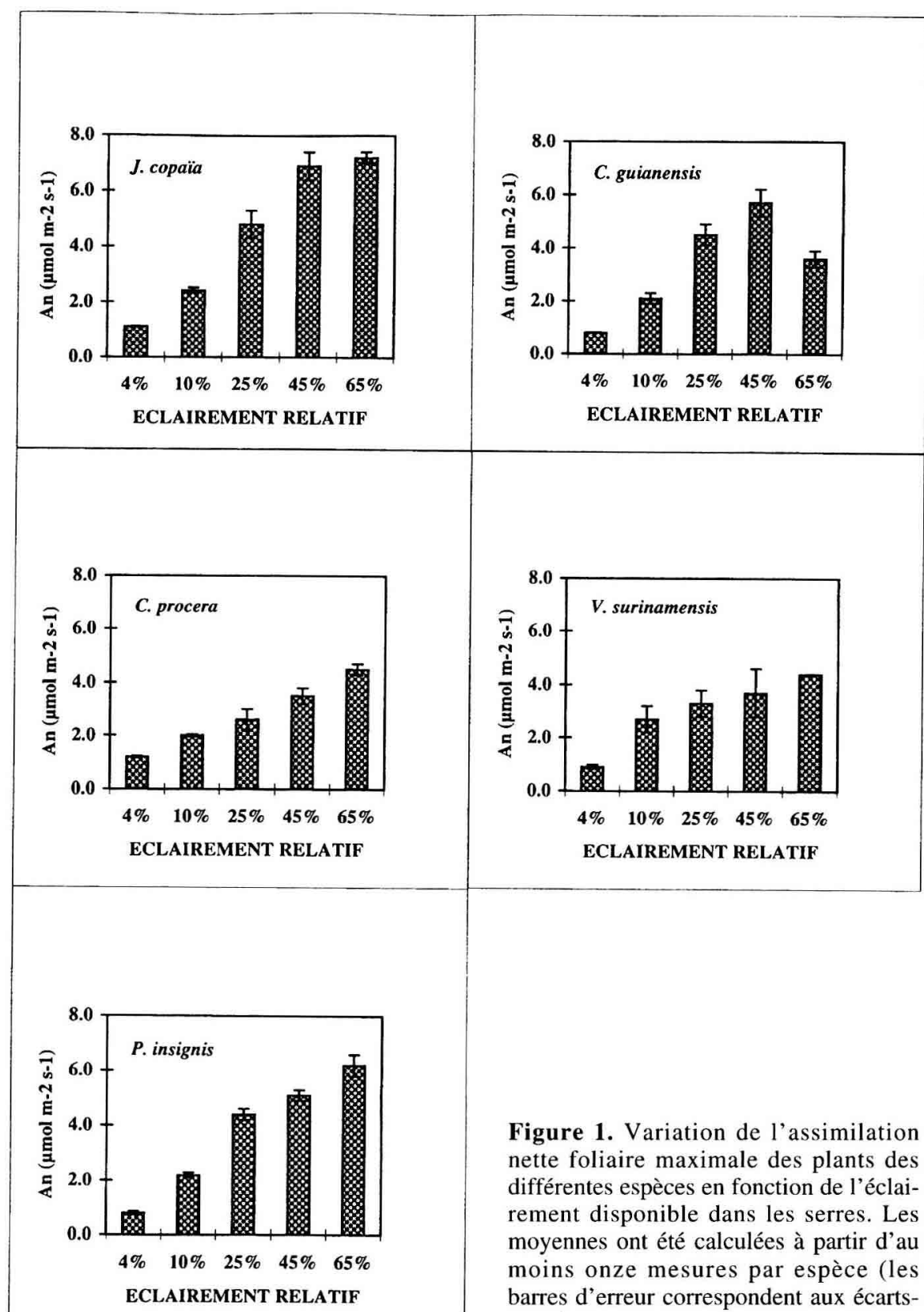

Figure 1. Variation de l'assimilation nette foliaire maximale des plants des différentes espèces en fonction de l'éclairement disponible dans les serres. Les moyennes ont été calculées à partir d'au moins onze mesures par espèce (les barres d'erreur correspondent aux écartstypes). 


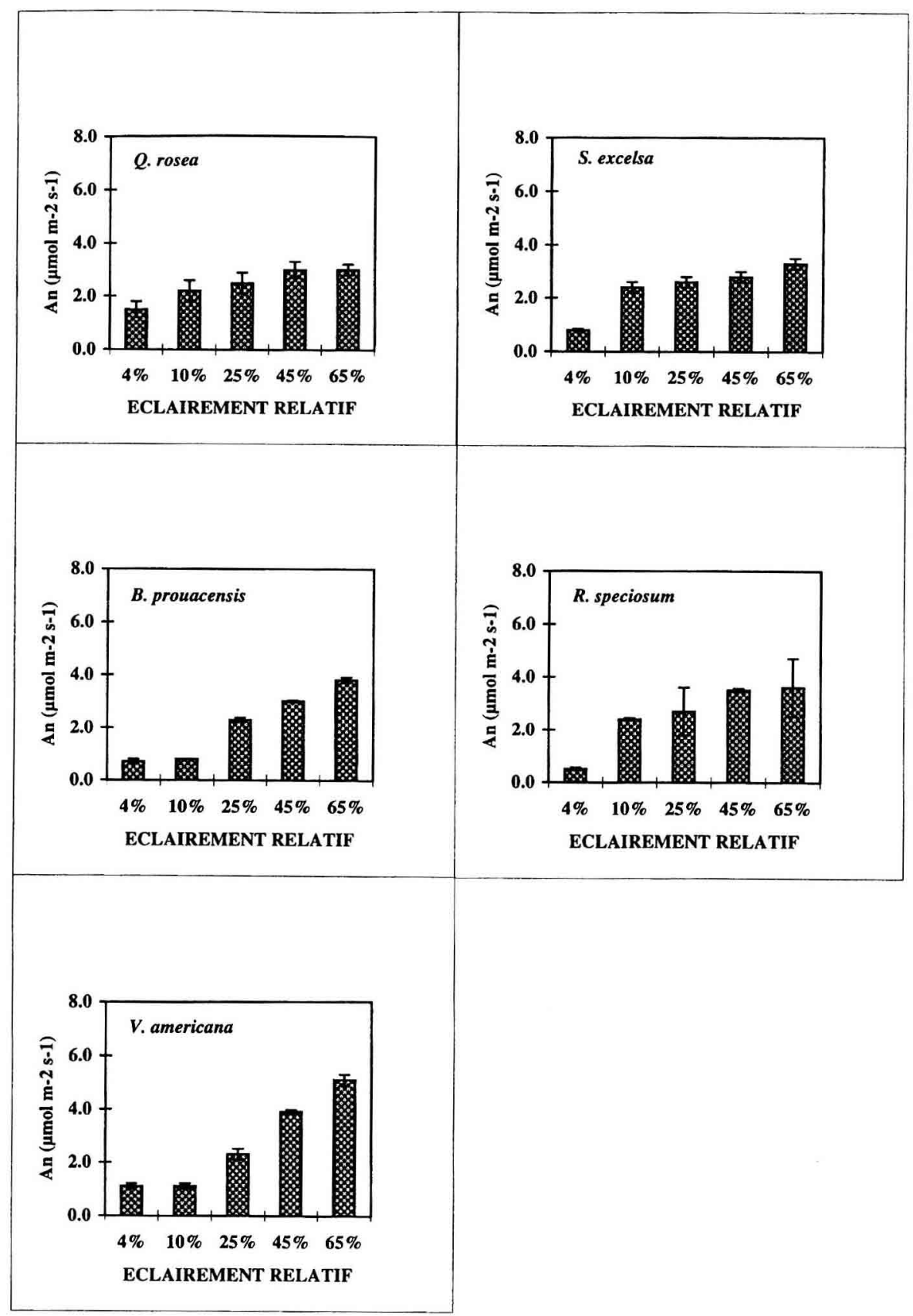


travaux antérieurs $[1,24,35]$. Les faibles taux de mortalité enregistrés seraient probablement liés à la plasticité de ces espèces vis-à-vis de leurs besoins en énergie lumineuse et à leur capacité à maintenir un bilan carboné positif ou nul. En effet, le bilan carboné constitue le facteur déterminant pour la survie des plantules dans un environnement de faible luminosité [41]. D'après les travaux de Augspurger [1], la plupart des plantules d'espèces tropicales, à l'exception de certaines Dipterocarpaceae, ont un faible taux de mortalité et un faible taux de croissance lorsqu'elles se développent à l'abri du rayonnement solaire direct. Toutefois, la durée de vie des plantules dans des conditions de faible éclairement reste inconnue de même que les effets de leur exposition brutale à la lumière (chablis).

\subsubsection{Croissance en hauteur, diamètre et volume de la tige}

Dans des conditions environnementales données, les processus de croissance dépendent des potentialités génétiques de chaque espèce. Ainsi, dans les conditions de fort éclairement $(65 \%$ du plein découvert), les plus forts et les plus faibles allongements moyens : $1,53 \mathrm{~m}$ et $0,18 \mathrm{~m}$ (figure 2) ont été respectivement observés chez Carapa guianensis (espèce opportuniste) et chez Bocoa prouacensis (espèce apparaissant tardivement au cours de la succession). Les différences de hauteur entre espèces varient d'un facteur 1 à 8,5 .

Les données collectées ont permis de définir globalement l'optimum d'éclairement relatif pour la croissance en hauteur des différentes espèces. Cet optimum se situerait entre 10 et $45 \%$ et plutôt proche de $25 \%$ du plein découvert pour la plupart des espèces. Les données relatives aux allongements ont aussi permis d'identifier quatre types de comportement de croissance en fonction du niveau d'éclairement (figure 2) :

- les espèces chez lesquelles la vitesse de croissance augmente avec l'éclairement puis se stabilise à partir de $25 \%$

Tableau I. Liste et densité du bois de l'arbre adulte des espèces étudiées.

\begin{tabular}{lll}
\hline Groupe écologique et Nom des espèces & Famille & $\begin{array}{l}\text { Densité du bois*. } \\
\text { (arbre adulte) }\end{array}$ \\
\hline
\end{tabular}

Pionniers ou héliophiles.

Jacaranda copaia (Aublet) D. Don

Opportunistes ou semi-tolérants.

Carapa guianensis Aublet

Carapa procera $\mathrm{CDC}$

Virola surinamensis (Rolander) Warburg

Platonia insignis Martins

Qualea rosea Aublet

Sterculia excelsa Martins

Tolérants ou sciaphiles.

Bocoa prouacensis Aublet

Recordoxylon speciosum (Benoist) Norm.

Vouacapoua americana Aublet

$\begin{array}{ll}\text { Bignoniaceae } & 0,40 \\ \text { Meliaceae } & 0,70 \\ \text { Meliaceae } & 0,70 \\ \text { Myristicaceae } & 0,48 \\ \text { Clusiaceae } & 0,85 \\ \text { Vochysiaceae } & 0,70 \\ \text { Sterculiaceae } & 0,60 \\ & \\ \text { Casalpiniaceae } & 1,22 \\ \text { Casalpiniaceae } & 0,95 \\ \text { Casalpiniaceae } & 0,92\end{array}$

\footnotetext{
* Sources : $[16,19] ;$ CTFT $=$ Centre Technique Forestier Tropical dont la nouvelle appellation est CiradForêt (Centre de coopération Internationale en Recherche Agronomique pour le Développement, Département forestier).
} 
d'éclairement relatif : Bocoa prouacensis, Carapa procera, Qualea rosea, Recordoxylon speciosum, Sterculia excelsa et Vouacapoua americana ;

- l'espèce chez laquelle la vitesse de croissance augmente avec l'éclairement, se stabilise pour une large plage d'éclairement relatif puis diminue lorsque l'éclairement relatif est supérieur à $45 \%$ : Virola surinamensis ;

- les espèces dont la vitesse de croissance, après une phase ascendante, est progressivement ralentie par l'augmentation de l'éclairement relatif : Carapa guianensis et Platonia insignis ;

- l'espèce qui présente un optimum de croissance nettement centré autour d'une valeur d'éclairement relatif : Jacaranda copaia (forte vitesse de croissance à $25 \%$ du plein découvert).

La croissance en diamètre de la plupart des espèces suit à peu près celle de la hauteur.

Les graphiques de la figure 3 représentent l'évolution de l'indice de volume de trois espèces durant la période d'étude. Il s'agit de Bocoa prouacensis, Carapa guianensis et Jacaranda copaia qui ont été choisies pour leurs comportements de croissance très typés. L'analyse de ces graphiques montre qu'il n'y a pas d'arrêt de croissance perceptible de façon significative quelle que soit l'espèce et la période de l'année. Il en est de même pour toutes les autres espèces. D'une façon générale, les effets significatifs de la lumière sur la croissance en volume ne sont perceptibles qu'environ six mois après la plantation. Les optima de croissance sont observés (tableau II) pour des éclairements relatifs de :

$25 \%$ pour Carapa guianensis et Jacaranda copaia ;

$45 \%$ pour Platonia insignis, Qualea rosea, Recordoxylon speciosum et Virola surinamensis ;
$65 \%$ pour Bocoa prouacensis, Carapa procera et Sterculia excelsa.

L'espèce Vouacapoua americana aurait des optima allant de 25 à $65 \%$.

\subsubsection{Croissance en biomasse totale et en surface foliaire}

Les graphiques de la figure 4 montrent les variations moyennes de production de biomasse totale (biomasses aérienne et souterraine) de trois espèces Bocoa prouacensis, Carapa guianensis et Jacaranda copaia en fonction de l'éclairement. À fort éclairement, la plus forte biomasse totale (286 $\mathrm{g}$ de matière sèche par plant) a été produite par Carapa guianensis et la plus faible production ( $8 \mathrm{~g}$ de matière sèche par plant) a été enregistrée chez Bocoa prouacensis. Différents comportements ont été notés chez les espèces étudiées. La similarité de comportement de production en fonction de l'éclairement a permis les regroupements suivants (tableau II) :

- les espèces chez lesquelles la production de biomasse augmente régulièrement avec l'éclairement relatif comme Bocoa prouacensis, Carapa procera et Sterculia excelsa ;

- les espèces dont la production de biomasse semble se stabiliser au-delà d'une certaine valeur d'éclairement comme Qualea rosea, Recordoxylon speciosum et Virola surinamensis ;

- les espèces chez lesquelles la formation de biomasse est ralentie au-delà de l'éclairement optimal comme Carapa guianensis, Platonia insignis et Vouacapoua americana (respectivement 25,25 et $45 \%$ pour ces espèces) ;

- l'espèce chez laquelle l'optimum de production est fortement centré sur une valeur de l'éclairement (25\%) : Jacaranda copaia.

Les graphiques de la figure 4 représentent les variations de la surface foliaire moyenne par plant de trois espèces Bocoa prouacensis, Carapa guianensis et 


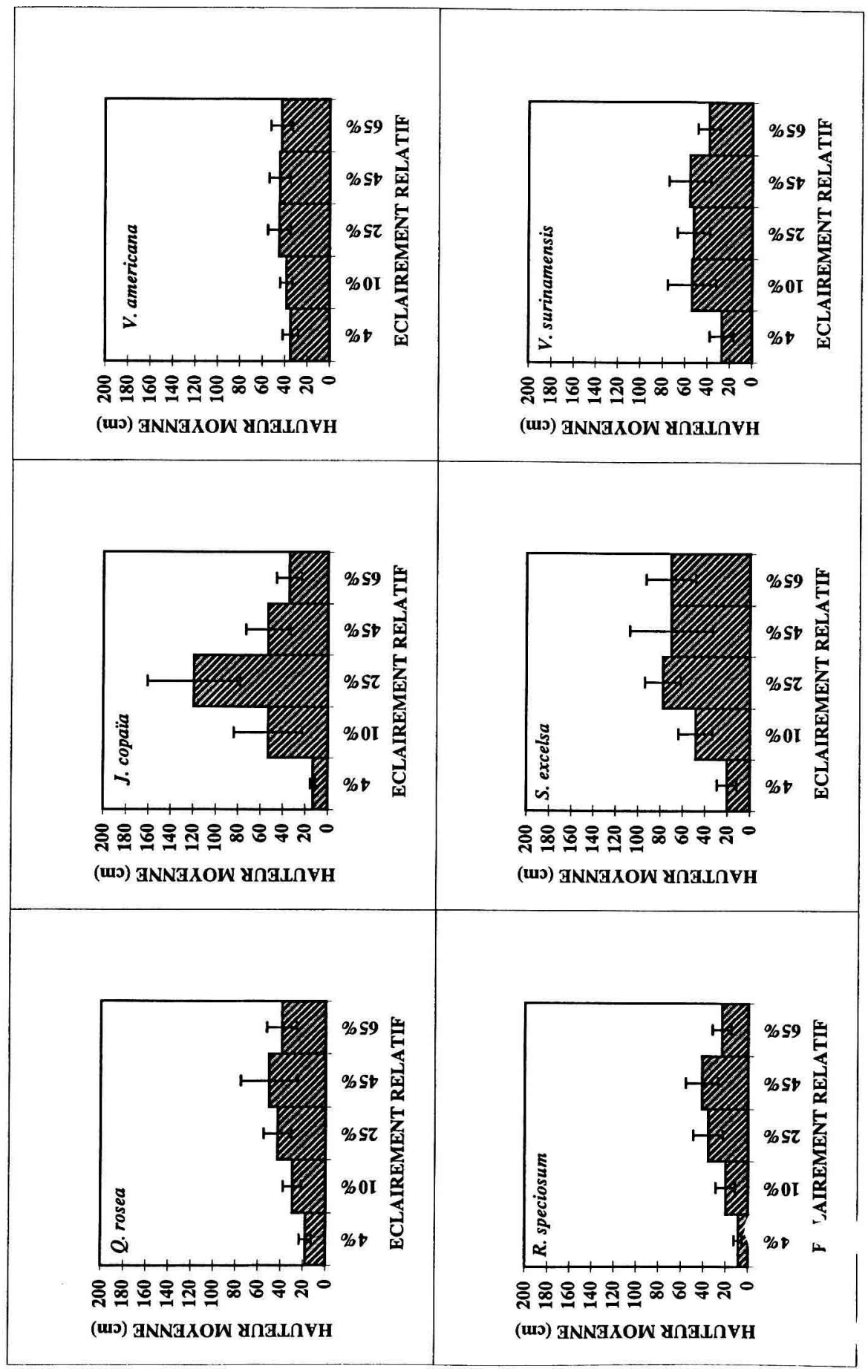




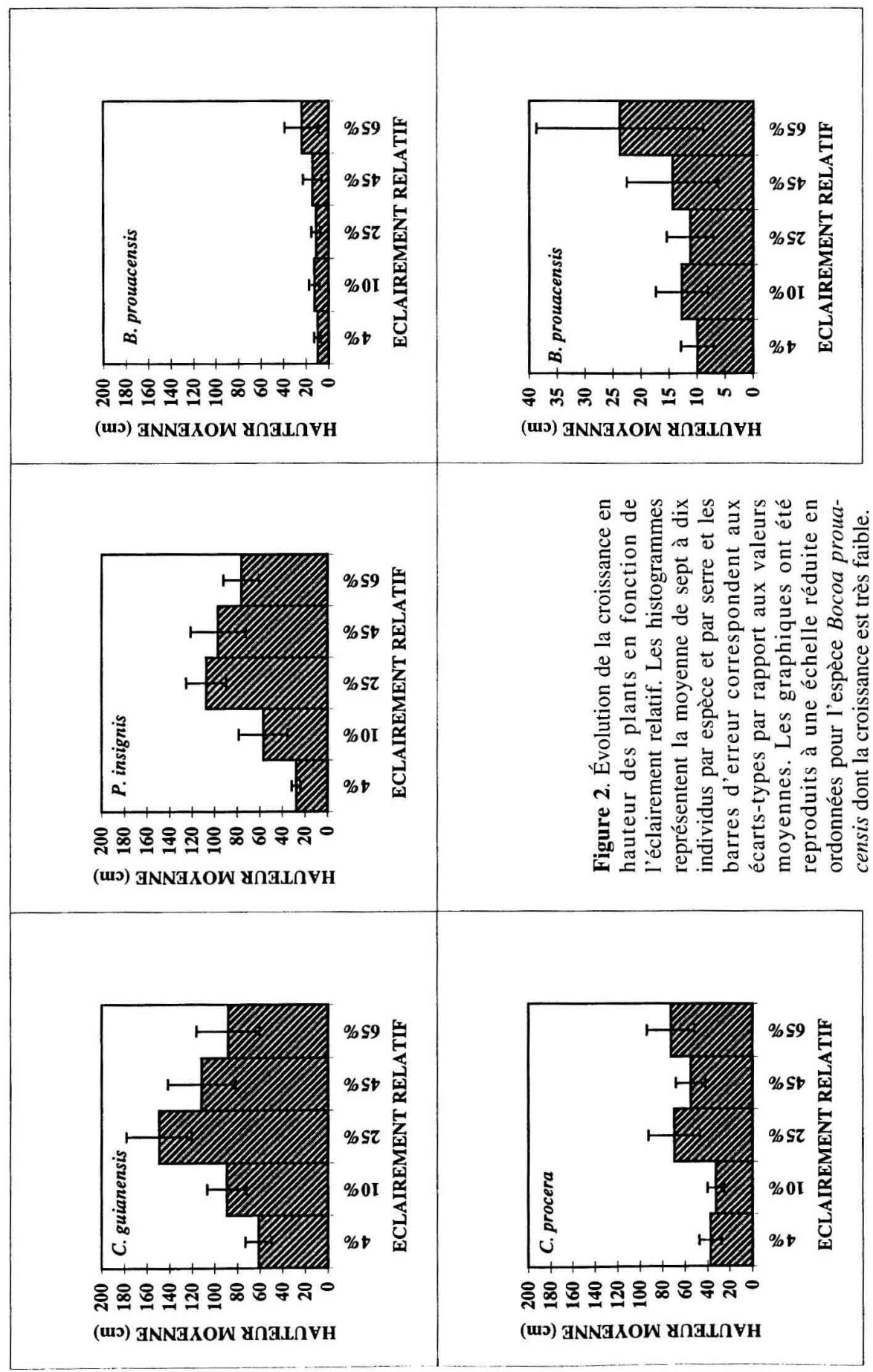


Tableau II. Effets des régimes lumineux sur les valeurs de surface foliaire, de biomasse totale et d'indice de volume des différentes espèces (Données collectées 701 jours après la plantation).

\begin{tabular}{|c|c|c|c|c|c|}
\hline $\begin{array}{l}\text { R lumineux } \\
\text { Espèces }\end{array}$ & $4 \%$ & $10 \%$ & $25 \%$ & $45 \%$ & $65 \%$ \\
\hline \multicolumn{6}{|l|}{ B. prouacensis } \\
\hline$S$ foliaire $\left(\mathrm{cm}^{2}\right)$ & 144,6 & 244,8 & 139,7 & 359,1 & 444,8 \\
\hline E type $\left(\mathrm{cm}^{2}\right)$ & 66,8 & 165,3 & 104,6 & 241,1 & 243,9 \\
\hline B totale $(\mathrm{g})$ & 3,1 & 8,5 & 7,6 & 11,5 & 15,2 \\
\hline E type (g) & 1,6 & 6,5 & 3,5 & 7,5 & 8,1 \\
\hline I volume $\left(\mathrm{cm}^{3}\right)$ & 113 & 224 & 194 & 333 & 835 \\
\hline E type $\left(\mathrm{cm}^{3}\right)$ & 50 & 190 & 138 & 339 & 799 \\
\hline \multicolumn{6}{|l|}{ C. guianensis } \\
\hline$S$ foliaire $\left(\mathrm{cm}^{2}\right)$ & 1654,4 & 4356,2 & 9274,3 & 6841,4 & 3369,6 \\
\hline E type $\left(\mathrm{cm}^{2}\right)$ & 511,0 & 1635,9 & 3367,2 & 3328,2 & 1257,8 \\
\hline B totale (g) & 23,6 & 86,6 & 257,7 & 222,1 & 177,4 \\
\hline E type (g) & 6,5 & 29 & 88,6 & 95,1 & 58,1 \\
\hline I volume $\left(\mathrm{cm}^{3}\right)$ & 5433 & 18557 & 64849 & 42624 & 32406 \\
\hline E type $\left(\mathrm{cm}^{3}\right)$ & 2769 & 8112 & 33984 & 23790 & 15908 \\
\hline \multicolumn{6}{|l|}{ P. insignis } \\
\hline$S$ foliaire $\left(\mathrm{cm}^{2}\right)$ & 463,9 & 2099,4 & 4679,1 & 3672,6 & 1780,7 \\
\hline E type $\left(\mathrm{cm}^{2}\right)$ & 199,9 & 1292,8 & 2041,4 & 1628,9 & 572,6 \\
\hline B totale $(\mathrm{g})$ & 14,6 & 56,7 & 174,6 & 265 & 149,6 \\
\hline E type (g) & 4,5 & 31,3 & 46,3 & 111,4 & 42,1 \\
\hline I volume $\left(\mathrm{cm}^{3}\right)$ & 794 & 6436 & 23083 & 31085 & 19482 \\
\hline E type $\left(\mathrm{cm}^{3}\right)$ & 215 & 4627 & 8082 & 16770 & 3390 \\
\hline \multicolumn{6}{|l|}{ C. procera } \\
\hline$S$ foliaire $\left(\mathrm{cm}^{2}\right)$ & 1513,7 & 1166,7 & 4509,1 & 2201,7 & 3739,9 \\
\hline E type $\left(\mathrm{cm}^{2}\right)$ & 1033,1 & 818,4 & 1714,0 & 1209,3 & 1832,8 \\
\hline $\mathrm{B}$ totale $(\mathrm{g})$ & 18,4 & 26,6 & 126 & 93,5 & 184 \\
\hline E type (g) & 8 & 7,2 & 43,2 & 33 & 78,5 \\
\hline I volume $\left(\mathrm{cm}^{3}\right)$ & 2610 & 3762 & 24716 & 17388 & 36600 \\
\hline E type $\left(\mathrm{cm}^{3}\right)$ & 1169 & 1814 & 11682 & 6736 & 23721 \\
\hline \multicolumn{6}{|l|}{ S. excelsa } \\
\hline$S$ foliaire $\left(\mathrm{cm}^{2}\right)$ & 602,2 & 2766,4 & 2955,4 & 3023,1 & 3093,9 \\
\hline E type $\left(\mathrm{cm}^{2}\right)$ & 237,8 & 1125,7 & 529,5 & 2045,5 & 2271,3 \\
\hline B totale $(\mathrm{g})$ & 7 & 47,4 & 79,2 & 81,8 & 144,8 \\
\hline E type (g) & 4,9 & 17,6 & 25,3 & 52,5 & 107,3 \\
\hline I volume $\left(\mathrm{cm}^{3}\right)$ & 861 & 8585 & 16428 & 19903 & 28693 \\
\hline E type $\left(\mathrm{cm}^{3}\right)$ & 1049 & 4886 & 7392 & 13633 & 30223 \\
\hline \multicolumn{6}{|l|}{ Q. rosea } \\
\hline$S$ foliaire $\left(\mathrm{cm}^{2}\right)$ & 330,4 & 2259,6 & 3360,2 & 2699,3 & 2232,7 \\
\hline E type $\left(\mathrm{cm}^{2}\right)$ & 174,2 & 1402,2 & 1390,4 & $1250, \mathrm{I}$ & 468,4 \\
\hline B totale $(\mathrm{g})$ & 2,9 & 21,3 & 61,3 & 60,7 & 50,3 \\
\hline E type (g) & 1,6 & 5,3 & 9,1 & 25,9 & 15,2 \\
\hline I volume $\left(\mathrm{cm}^{3}\right)$ & 209 & 1104 & 4417 & 8196 & 4048 \\
\hline E type $\left(\mathrm{cm}^{3}\right)$ & 109 & 604 & 2978 & 4810 & 2220 \\
\hline
\end{tabular}


V. americana

$S$ foliaire $\left(\mathrm{cm}^{2}\right)$

E type $\left(\mathrm{cm}^{2}\right)$

B totale $(\mathrm{g})$

E type $(\mathrm{g})$

I volume $\left(\mathrm{cm}^{3}\right)$

E type $\left(\mathrm{cm}^{3}\right)$

R. speciosum

$\mathrm{S}$ foliaire $\left(\mathrm{cm}^{2}\right)$

E type $\left(\mathrm{cm}^{2}\right)$

B totale $(\mathrm{g})$

E type (g)

I volume $\left(\mathrm{cm}^{3}\right)$

E type $\left(\mathrm{cm}^{3}\right)$

\section{V. surinamensis}

$\mathrm{S}$ foliaire $\left(\mathrm{cm}^{2}\right)$

E type $\left(\mathrm{cm}^{2}\right)$

B totale $(\mathrm{g})$

E type (g)

I volume $\left(\mathrm{cm}^{3}\right)$

E type $\left(\mathrm{cm}^{3}\right)$

J. copaia

$\mathrm{S}$ foliaire $\left(\mathrm{cm}^{2}\right)$

E type $\left(\mathrm{cm}^{2}\right)$

B totale $(\mathrm{g})$

E type $(\mathrm{g})$

I volume $\left(\mathrm{cm}^{3}\right)$

E type $\left(\mathrm{cm}^{3}\right)$

$\begin{array}{cc}1023,4 & 2059,1 \\ 479,1 & 302,2 \\ 18,0 & 37,2 \\ 6,6 & 6,9 \\ 1713 & 2594 \\ 809 & 739\end{array}$

209,0

\section{4,5}

1,2

0,9

41

31

1324,5
1167,4
11,7
12,9
766
895

$$
236,3
$$

197,7

5,3

2,5

1346

965

1712,8
1652,9
40,9
28,4
8878
6560

347,7
232,7
3
1
201
57

2122,7
1337,9
30,1
18,7
8213
8246

8213
8246
10265,4
5426,6
196,3
114,2
46996
34398

$\begin{array}{cc}1740,7 & 1048,0 \\ 589,8 & 494,4 \\ 48,9 & 33,6 \\ 10,9 & 10,6 \\ 4302 & 4045 \\ 1391 & 1829\end{array}$

$\begin{array}{cc}3083,0 & 1866,0 \\ 2063,3 & 859,6 \\ 46,7 & 36,2 \\ 35,3 & 26,3 \\ 3931 & 2138 \\ 3925 & 2690\end{array}$

$\begin{array}{rr}2437,9 & 1642,5 \\ 608,7 & 470,7 \\ 101,4 & 91,1 \\ 26,3 & 21,7 \\ 18518 & 11381 \\ 7972 & 3272\end{array}$

$\begin{array}{cc}2279,2 & 1069,6 \\ 415,2 & 531,2 \\ 66,3 & 48,1 \\ 19,6 & 13,3 \\ 9860 & 6107 \\ 3461 & 3692\end{array}$

Jacaranda copaia en fonction de l'éclairement relatif. Les plus fortes valeurs moyennes de surface foliaire ont été observées chez les espèces Jacaranda copaia $\left(1.03 \mathrm{~m}^{2}\right.$ à $25 \%$ d'éclairement relatif) et Carapa guianensis $\left(0.93 \mathrm{~m}^{2}\right.$ à $25 \%$ d'éclairement relatif). L'examen des profils des courbes de variation de la surface foliaire et de celle des biomasses totales révèle que l'évolution de ces deux variables est identique. Le regroupement des différentes espèces en fonction de la croissance foliaire en tenant compte de l'éclairement relatif est à l'image de celui réalisé précédemment pour la biomasse totale (tableau II).

\subsection{Relations entre biomasse totale et surface foliaire}

La grande majorité des végétaux terrestres évoluent dans les deux types de milieu aérien et édaphique. Les variables de ces milieux pouvant potentiellement influencer la croissance foliaire sont la température, l'éclairement, la concentration en dioxyde de carbone de l'air et l'eau, les substances nutritives, la température du sol. Du fait de la grande sensibilité de la croissance foliaire à ces variables environnementales, la production et la croissance des plants sont fréquemment sujettes à des limitations [49]. 


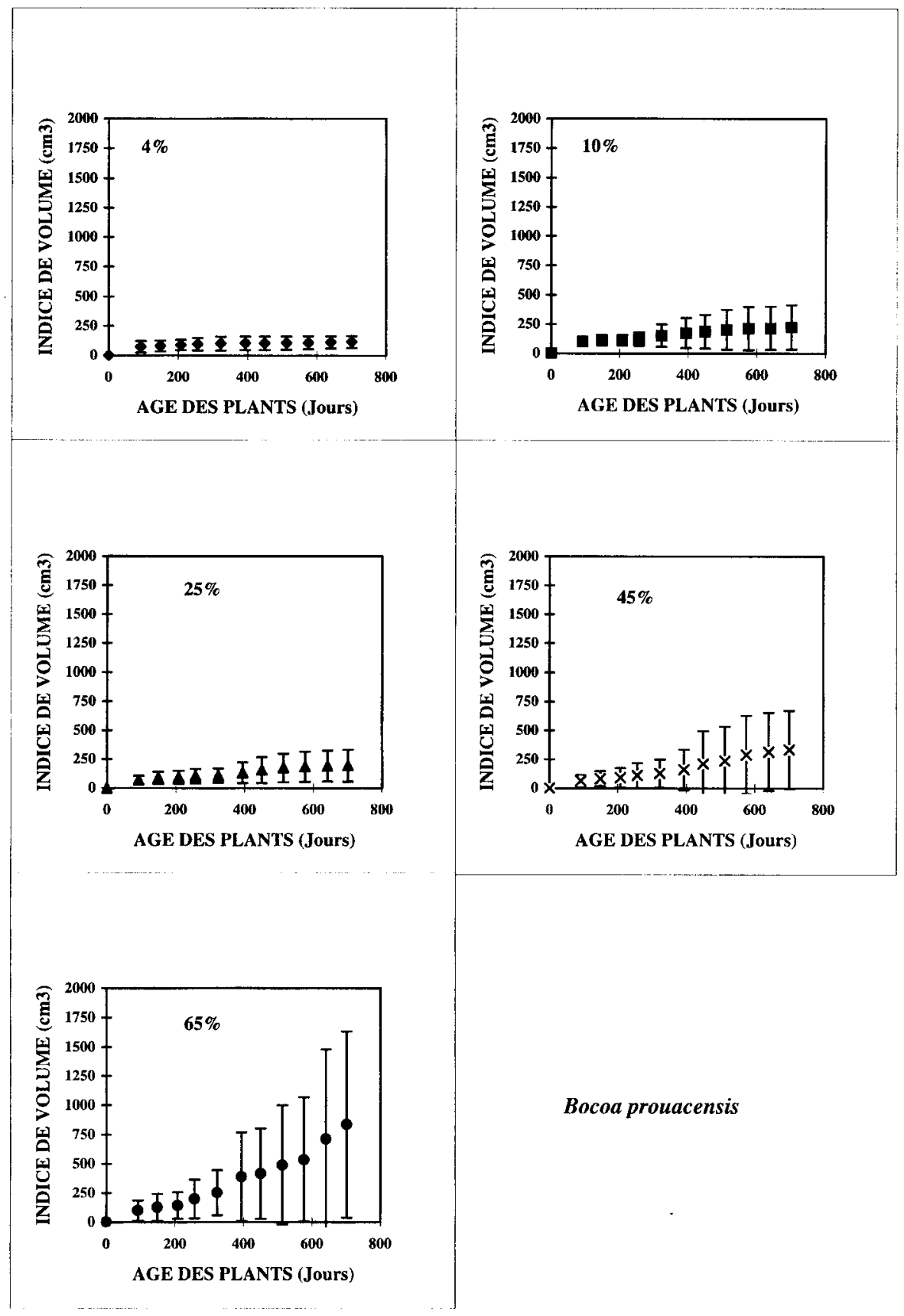



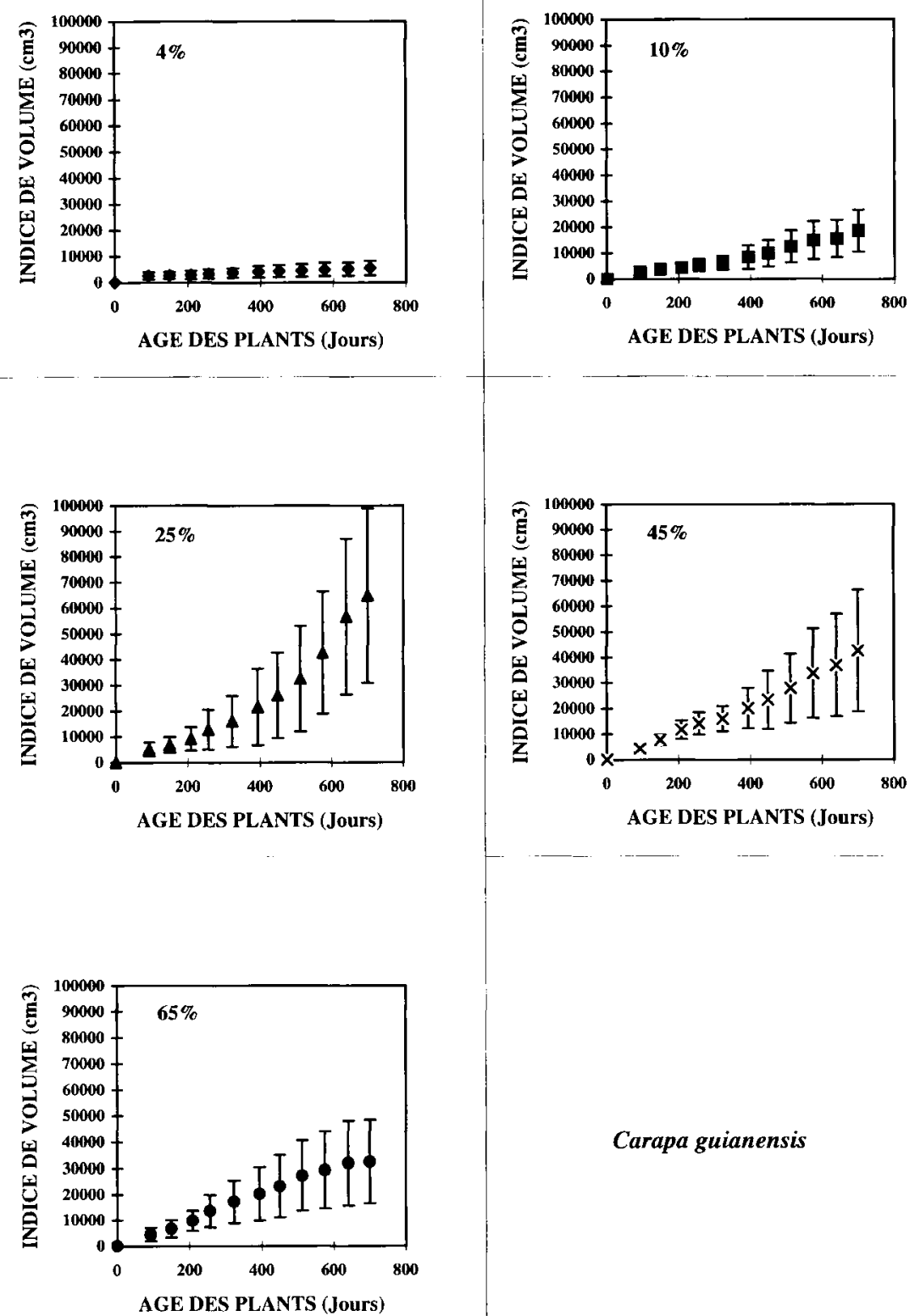

Carapa guianensis 


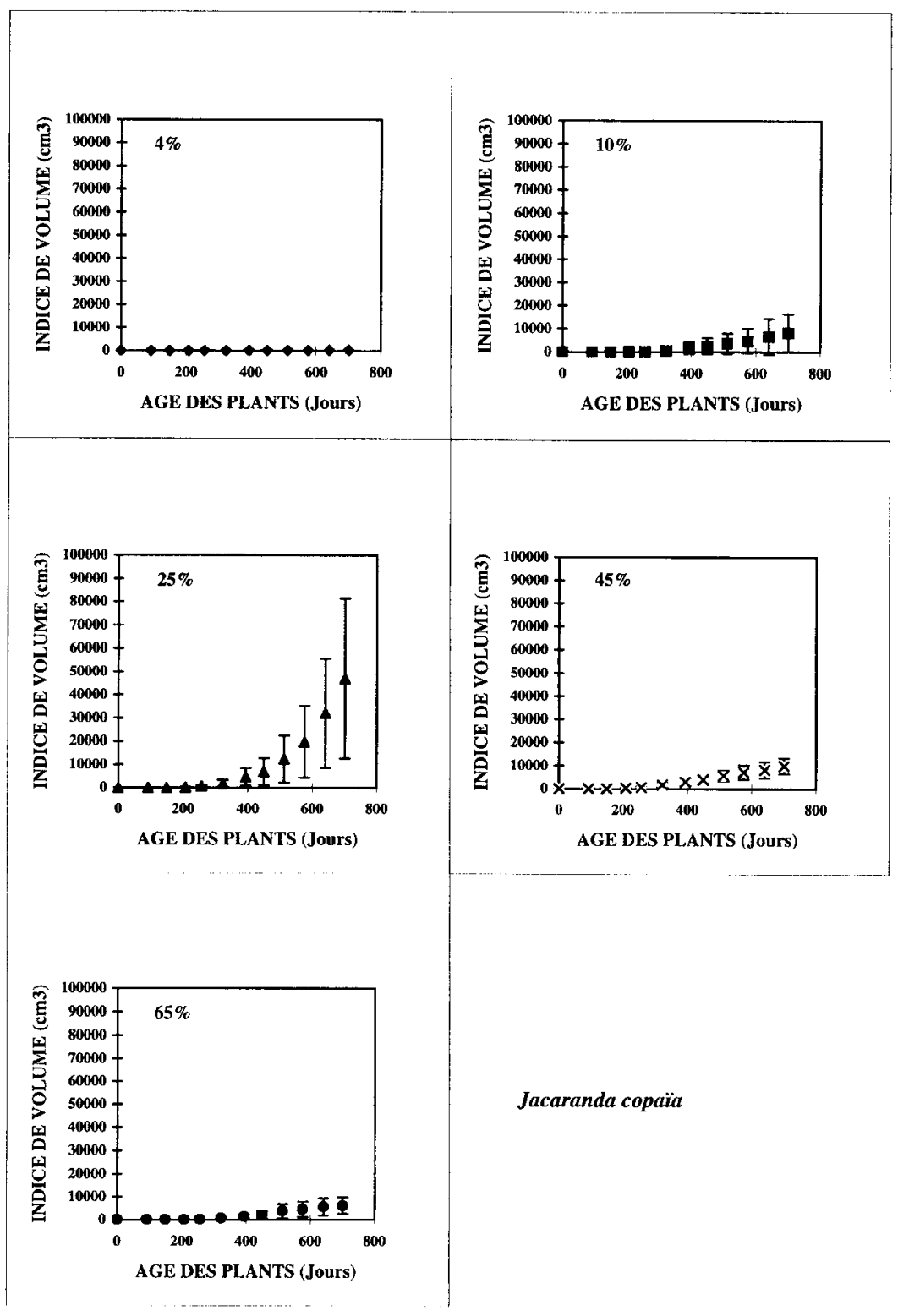

Figure 3. Évolution de l'indice de volume (hd $\left.{ }^{2}\right)$ des plants de trois espèces Bocoa prouacensis, Carapa guianensis et Jacaranda copaia en fonction du temps (nombre de jours après plantation). Les points représentent la moyenne de sept à dix individus par espèce et par serre et les barres d'erreur correspondent aux écarts-types par rapport aux valeurs moyennes. Les graphiques ont été reproduits à une échelle réduite en ordonnée pour l'espèce Bocoa prouacensis dont l'indice foliaire est très faible. 


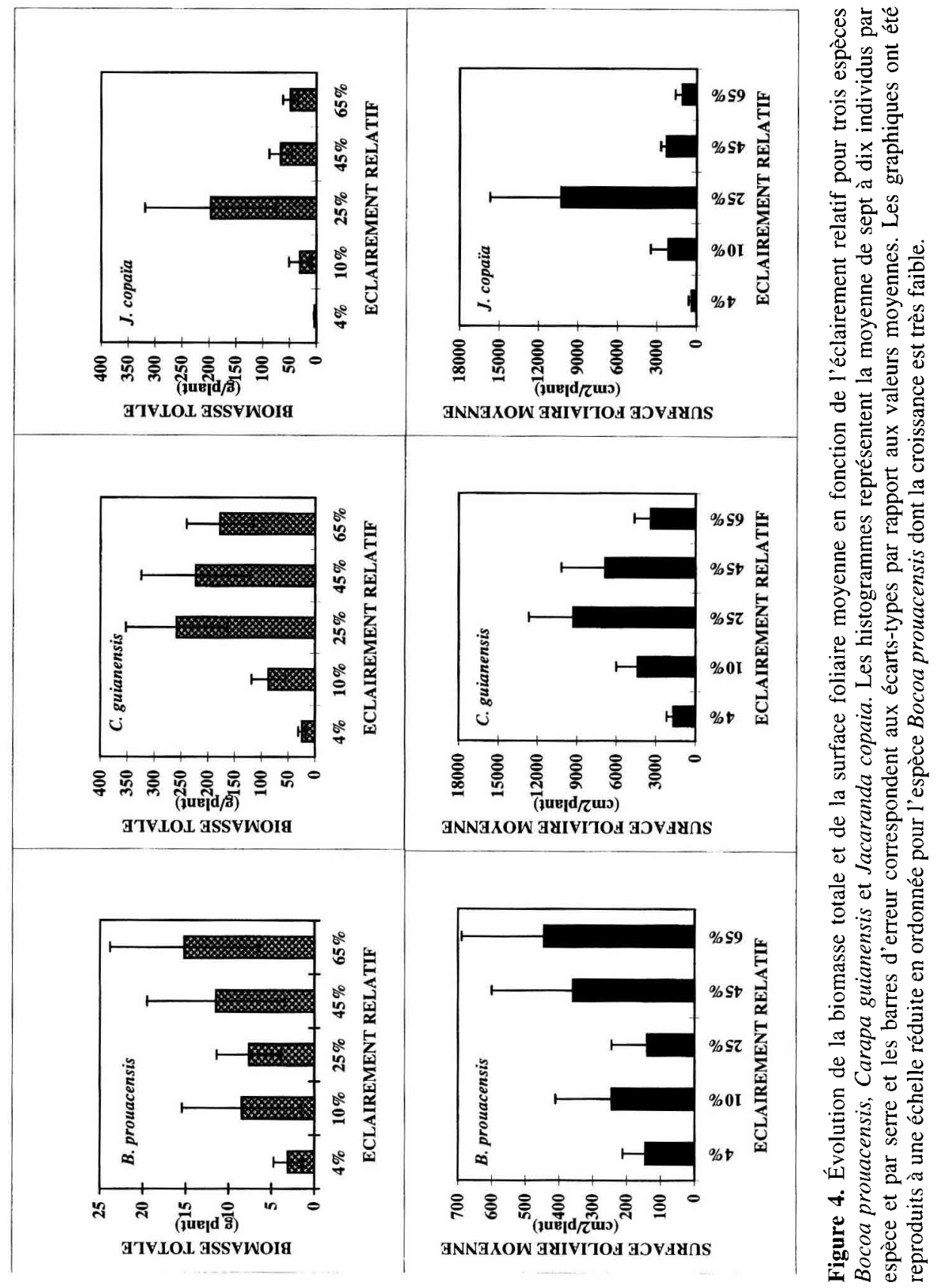


La diminution graduelle de la surface foliaire observée chez la plupart des espèces, après l'optimum de croissance alors que le taux d'assimilation nette foliaire se maintient ou continue d'augmenter, pourrait être attribuée aux effets conjugués des différentes variables de l'environnement et plus particulièrement aux effets d'un déficit hydrique sur la croissance [25]. À $25 \%$ d'éclairement relatif, les surfaces foliaires maximales de Jacaranda copaia et de Carapa guianensis sont proches d'un mètre carré et peuvent donc être à l'origine de transpiration journalière dépassant les $2 \mathrm{~L}$ d'eau. La croissance de ces plants et de ceux soumis à des régimes lumineux supérieurs à $25 \%$ du plein éclairement serait davantage affectée par l'insuffisance des apports d'eau. En revanche, les plants et espèces qui développent une surface foliaire modérée et/ou qui régulent suffisamment leurs flux transpiratoires pourraient bénéficier davantage de supplément de lumière pour augmenter leur production de matière ; n'étant pas limités par la disponibilité en eau du sol. Dans ce travail comme dans la plupart des études conduites sur des plantes en pot, la quantité d'eau apportée par irrigation est en général sous-estimée [12] à cause du volume de sol dont disposent les plantes et par conséquent des risques d'engorgement d'eau. Dans les conditions d'éclairement relatif supérieur ou égal à $25 \%$, la vitesse de croissance maximale de ces plantes serait probablement affectée par des insuffisances d'eau auxquelles s'ajouteraient d'autres facteurs qui diminueraient de façon interactive l'efficience d'utilisation de l'eau [38].

\subsection{Relations entre assimilation nette foliaire et biomasse totale}

Les graphiques de la figure 5 illustrent le degré de dépendance entre assimilation nette foliaire maximale et production maximale de biomasse totale pour les espèces étudiées. À l'exception de Bocoa prouacensis et de Carapa procera, le taux d'assimilation nette foliaire maximale ne semble pas constituer un paramètre explicatif de la production de biomasse. Chez des espèces comme Carapa guianensis, Jacaranda copaia et Platonia insignis, les fortes valeurs d'assimilation nette foliaire ne se traduisent pas par une production élevée de matière sèche. La quantité de biomasse produite par rapport à la capacité d'assimilation nette foliaire est faible chez les espèces comme Bocoa prouacensis, Recordoxylon speciosum, Vouacapoua americana.

La croissance et l'accumulation de matière sèche d'une plante résulte de la production et de l'utilisation d'assimilats élaborés par chacune des feuilles. Or, dans le feuillage, on trouve des feuilles d'âge différent ; leur contribution au gain de carbone $[20,28]$ dépend de leur stade de développement, de leurs position et orientation par rapport à la lumière, qui dépend du port et du développement de la plante elle-même. De plus, chaque feuille est soumise, dans les conditions naturelles (en fonction de sa position dans le couvert), à des facteurs environnementaux (éclairement, température, humidité) plus ou moins fluctuants au cours de la journée puis de la saison $[34,53]$. La variabilité de la réponse foliaire est, en général, très importante et donc source d'erreurs d'évaluation [29].

L'analyse des résultats d'assimilation nette foliaire maximale en rapport avec la production totale de biomasse de chaque espèce pose un problème : l'assimilation nette foliaire est-elle corrélée à la production de biomasse? Dans de nombreux cas, comme dans ce travail, l'assimilation nette foliaire est très peu corrélée à la croissance et à la production de biomasse $[39,43]$. Cette faible corrélation serait liée à la fois aux caractéristiques foliaires et aux variations physiologiques et phé- 
nologiques qui se produisent dans les feuilles au cours du temps [4] et aussi à la différence des capacités de conversion de la lumière en assimilats utilisables pour la croissance puis de la transformation de ces assimilats en matière sèche.

Chez les espèces sciaphiles étudiées dans ce travail (Bocoa prouacensis, Recordoxylon speciosum, Vouacapoua americana), la densité du bois de l'arbre adulte est très élevée par rapport à celle observée chez les espèces des autres groupes écologiques (tableau I), le faible taux de conversion des assimilats en biomasse pourrait s'expliquer par des coûts de production élevés de matière sèche de forte densité [21,36]. Cette caractéristique semble être commune aux espèces appartenant au groupe écologique des espèces sciaphiles [1]. Les coûts de production de matière sèche en relation avec la densité du bois de l'arbre adulte ne semblent toutefois pas suffisants pour expliquer la variabilité des relations existant entre taux d'assimilation nette foliaire maximale et production maximale de biomasse totale observée chez les autres espèces.

L'étude de la dynamique démographique [35] induite par les traitements sylvicoles d'intensités variables sur la régénération naturelle en forêt tropicale humide de quelques unes des espèces utilisées dans ce travail a révélé des différences de comportements (indifférent, bénéfique ou défavorable) à la mise en lumière. Selon cet auteur, la mise en lumière consécutive aux traitements sylvicoles aurait un effet favorable sur l'installation des semis de Bocoa prouacensis. Cette observation in situ (bilan d'inventaires effectués tous les deux ans de 1986 à 1992 après traitements sylvicoles) corrobore les résultats obtenus dans ce travail sur la croissance de jeunes plants de cette espèce. Bien que cette espèce soit connue pour ses caractéristiques de plante d'ombre $[8,19]$, la mise en lumière favo- riserait la production d'assimilats qui seraient utilisés pour la croissance et le développement.

Une nette réduction d'effectifs des semis de l'espèce Qualea rosea [35] liée à l'application des traitements sylvicoles a été observée. La mise en lumière brutale serait défavorable à la survie des semis alors qu'elle serait bénéfique pour les stades plus avancés (jeunes tiges) en terme de vitesse de croissance et de recrutement dans des classes de diamètres plus importants [35]. Les jeunes plants de cette espèce supportent d'autant mieux la mise en lumière intensive qu'ils ont atteint, avant la perturbation, un certain seuil de développement. Les résultats obtenus dans de ce travail ne reflètent pas les observations de terrain puisque la croissance en biomasse observée aux forts éclairements nest pas significativement différente de celle des conditions de croissance optimale.

Les comportements in situ [35] et en conditions de serre de Virola surinamensis sont assez proches de ceux de Qualea rosea.

La mise en lumière consécutive aux traitements sylvicoles d'intensité variable s'est traduite par une explosion démographique de semis de Jacaranda copaia, espèce quasi absente en régénération avant perturbation [35]. Une très nette diminution (bilan négatif) d'effectifs parmi les semis a été enregistrée pour les traitements de forte intensité [35]. Ces résultats semblent refléter les comportements des individus de cette espèce cultivés dans les conditions de ce travail. La croissance au stade jeune semble bien être plus forte dans une plage étroite de lumière atténuée.

Une fluctuation importante d'effectifs des semis de Sterculia excelsa a été observée [35]. Les traitements sylvicoles de forte intensité ont été plutôt défavorables à cette espèce dont les semis sont très peu abondants en régénération avant 


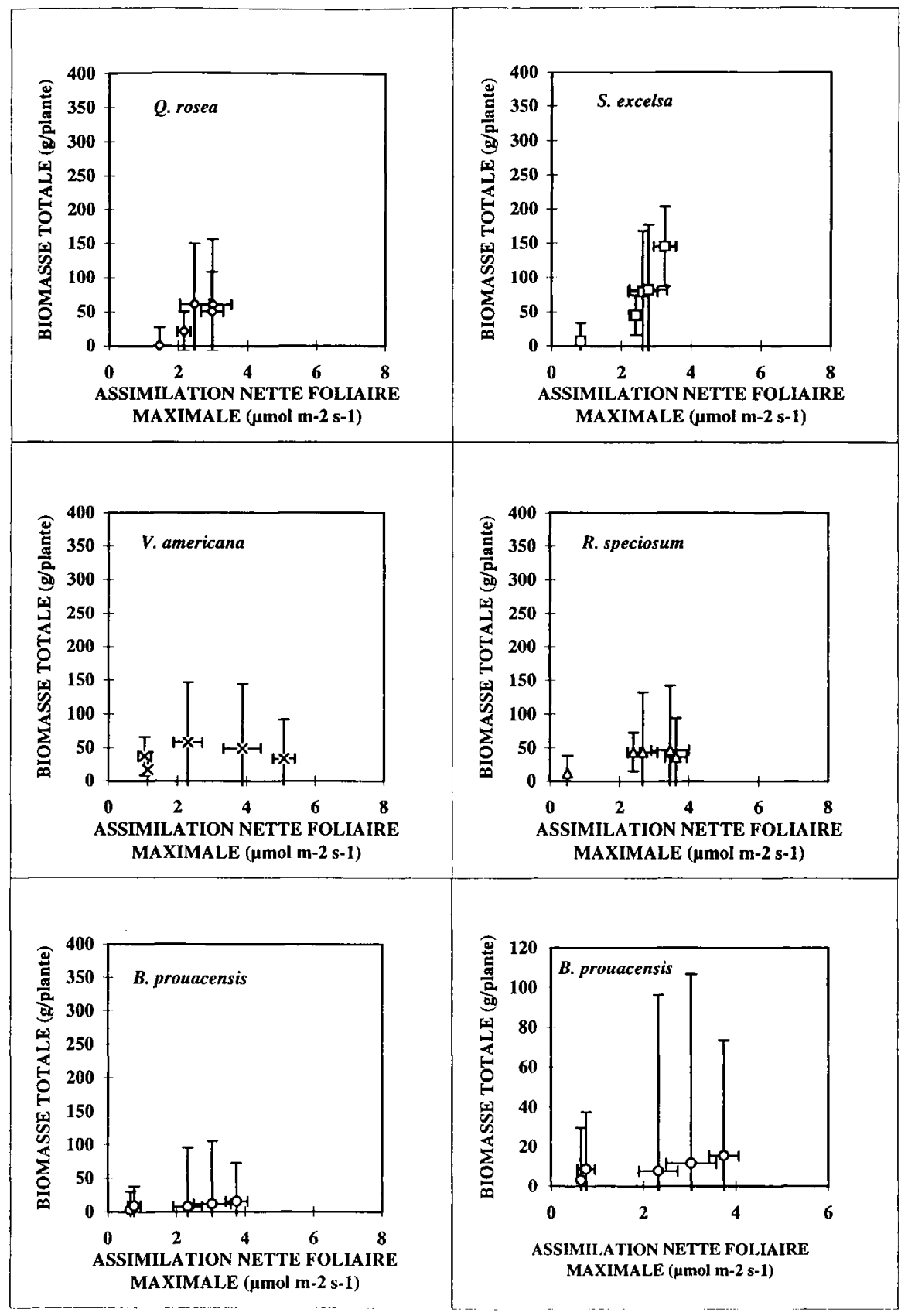



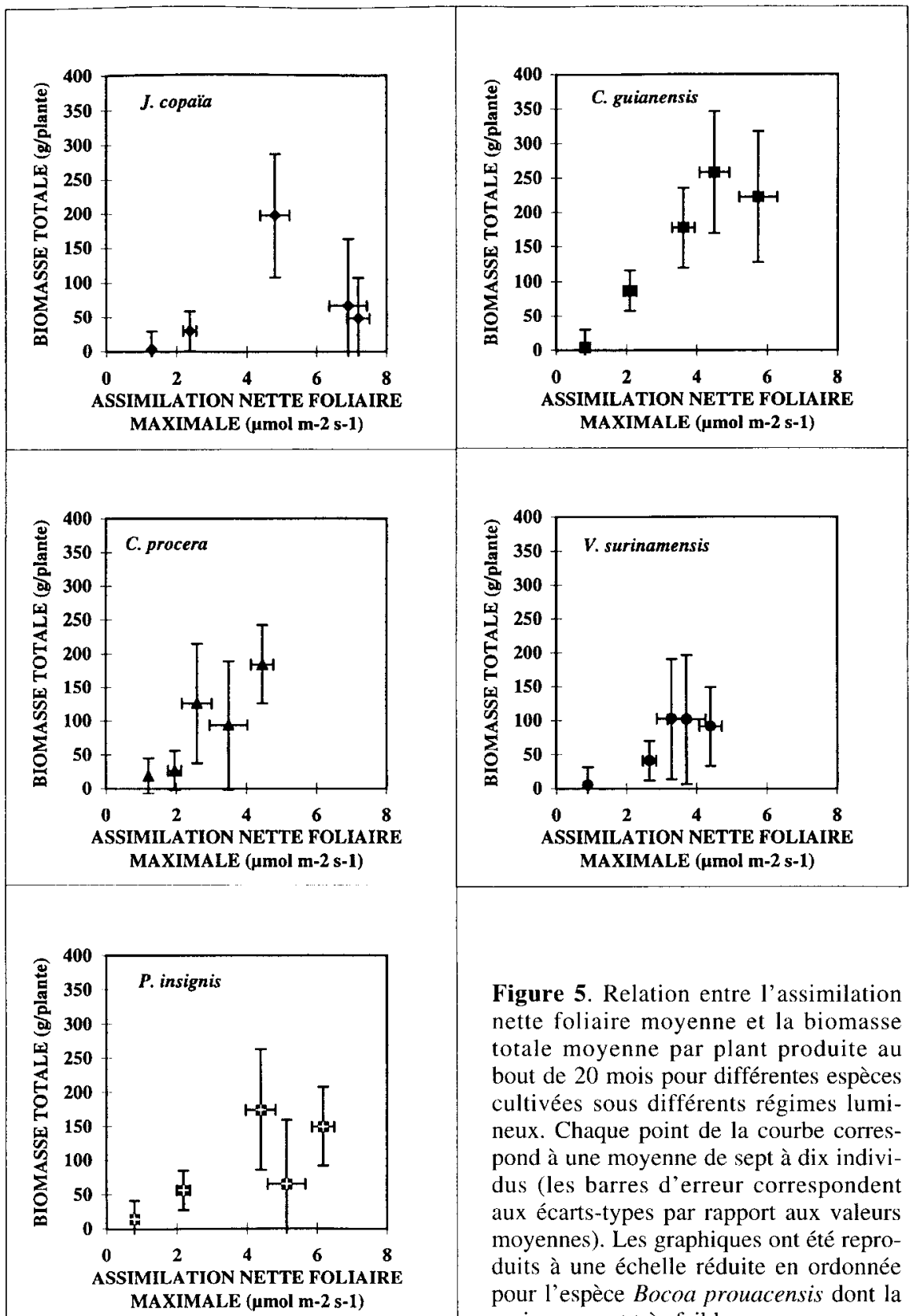

Figure 5. Relation entre l'assimilation nette foliaire moyenne et la biomasse totale moyenne par plant produite au bout de 20 mois pour différentes espèces cultivées sous différents régimes lumineux. Chaque point de la courbe correspond à une moyenne de sept à dix individus (les barres d'erreur correspondent aux écarts-types par rapport aux valeurs moyennes). Les graphiques ont été reproduits à une échelle réduite en ordonnée pour l'espèce Bocoa prouacensis dont la croissance est très faible. 
traitement. À l'opposé, les traitements de faible intensité ont été bénéfiques à l'espèce. Ces résultats de terrain sont cohérent avec les écart-types observés en étudiant les effets de l'éclairement disponible dans les serres sur la croissance en biomasse des jeunes plants de cette espèce.

Le comportement de terrain des espèces Vouacapoua americana et Carapa procera n'a pu être évalué à cause de leur taux de représentativité trop faible en régénération avant traitements sylvicoles [35]. Pour les mêmes raisons probablement, les espèces Platonia insignis et Recordoxylon speciosum n'ont pas été prises en compte dans les études d'impact des traitements sylvicoles sur la démographie de la régénération naturelle en forêt tropicale humide [35]. Les semenciers de Carapa guianensis seraient très peu représentés voire absents du dispositif expérimental de Paracou où ces études d'impact des traitements sylvicoles ont été conduits (Montpied, communication personnelle).

\section{CONCLUSION}

De ces études d'assimilation nette foliaire et de croissance des espèces soumises à différents niveaux d'éclairement, il ressort :

1) que dans des conditions d'éclairement relatif inférieur ou égal à $65 \%$, la plupart des espèces montrent une plasticité relativement grande pour leurs besoins en énergie lumineuse avec un optimum de croissance situé entre 10 et $45 \%$ du plein découvert ;

2) que paradoxalement l'optimum de croissance des espèces dites pionnières (colonisatrices des milieux ouverts) se situe en général aux environs de $25 \%$ du plein éclairement alors que les plantules de certaines espèces tolérantes à l'ombrage ont leur optimum de crois- sance à des éclairements plus élevés (supérieur ou égal à $65 \%$ du plein découvert) ;

3) qu'il n'y a pas de relations proportionnelles entre assimilation nette foliaire et croissance en biomasse totale chez la plupart des espèces étudiées ;

4) que la surface foliaire par plant constitue le facteur déterminant de la croissance en biomasse totale.

Par ailleurs, il a été observé que les perturbations du milieu (culture en pot et en conditions semi-contrôlées) n'ont pas empêché les jeunes plants d'être endomycorhizés comme les plants prélevés en forêt [9]. La présence de mycorhizes sur les racines des plants cultivés en pot est un élément important pour ce qui est de la représentativité des résultats [23].

Les résultats obtenus en conditions semi-contrôlées doivent être extrapolés avec précautions aux conditions naturelles. En effet, la qualité du rayonnement (longueur d'onde) reste différente de celle filtrée par le couvert forestier et peut affecter la morphologie et la physiologie des plantules $[46,47]$. La zone d'extension des racines est limitée par la taille des pots et les paramètres de concurrence sont modifiés. Toutefois, les études déjà menées en forêt tropicale humide [35] et dans les forêts tempérées [2] montrent l'importance et la fiabilité de l'utilisation des connaissances acquises sur les réponses à l'éclairement de jeunes arbres en sylviculture.

L'analyse globale des données recueillies sur le comportement de croissance des espèces étudiées a permis d'aboutir à des résultats en accord avec ceux de travaux antérieurs réalisés sur différentes espèces $[22,26,27]$. Ces auteurs ont mis en évidence, en réponse aux variations des facteurs du climat, une très forte variabilité interspécifique de la transpiration, de l'activité photosynthétique et de l'efficience photosynthétique d'utilisation de l'eau des arbres de la 
forêt tropicale humide tant en situation de forêt primaire qu'en plantation artificielle. Cette variabilité a également été observée parmi des essences d'intérêt commercial cultivées en serre-tunnels et sous différents régimes lumineux.

L'aptitude d'une plante à maintenir des optima de croissance en réponse à de larges plages d'éclairement pourrait constituer une caractéristique adaptative d'occupations sélectives du milieu. Les caractéristiques adaptatives favoriseraient la survie et le développement des espèces dans des environnements microclimatiques lumineux fluctuants du fait des structurations verticale et horizontale des écosystèmes forestiers tropicaux humides.

\section{ANNEXE 1 . \\ BRÈVE DESCRIPTION DES ESPÈCES UTILISÉES [8, 16, 35].}

\section{Bocoa prouacensis (Caesalpiniaceae)} se trouve le plus fréquemment en forêt primaire sur des terrains sains, souvent à flanc de collines. C'est une essence d'ombre qui possède une régénération peu abondante. Elle est de petite taille ; sa hauteur dépasse rarement $25 \mathrm{~m}$ et son diamètre est compris entre 50 et $70 \mathrm{~cm}$. Son houppier a une forme très irrégulière et une importance moyenne. Les feuilles sont simples et de dimensions très variables.

Carapa guianensis et Carapa procera (Meliaceae) sont des essences de demi lumière ( $C$. guianensis) ou de pleine lumière $(C$. procera) à régénération abondante. Elles se rencontrent le plus souvent sur les terrains humides, les bords de cours d'eau et parfois sur des collines. Ce sont de grands arbres pouvant atteindre 30 à $40 \mathrm{~m}$ de haut avec un diamètre compris entre 50 et $80 \mathrm{~cm}$. Son houppier est plus haut que large. Les feuilles sont alternes, composées paripennées et mesu- rent entre 15 et $35 \mathrm{~cm}$. Les folioles de $C$. procera se distinguent de ceux de $C$. guianensis par leur sommet très arrondi.

Jacaranda copaia (Bignoniaceae) est une essence de pleine lumière qui pousse de préférence dans les zones inondables et se contente de sols pauvres. C'est un arbre de petite taille qui mesure entre 16 et $20 \mathrm{~m}$ de haut pour un diamètre compris entre 50 et $80 \mathrm{~cm}$. Le feuillage est groupé à l'extrémité des branches et la cime est peu importante. L'essence possède des feuilles composées, bipennées, pouvant mesurer entre 35 et $40 \mathrm{~cm}$ de long. Elle est peu fréquente en régénération. Elle est généralement qualifiée de pionnière sans toutefois posséder le comportement héliophile typique [35].

Platonia insignis (Clusiaceae) se développe dans les forêts primaires en terrains sains. C'est une essence de demi-lumière à régénération abondante. Les feuilles sont simples, opposées, elliptiques, obtuses à la base et arrondies au sommet. Le fût cylindrique et droit de $25 \mathrm{~m}$ environ se termine par une cime très importante.

Qualea rosea (Vochysiaceae) est une essence de l'étage dominant en forêt qui pousse de préférence sur des sols fermes mais on le trouve aussi sur des sols marécageux. L'arbre dépasse rarement $30 \mathrm{~m}$ de haut avec un diamètre pouvant atteindre $100 \mathrm{~cm}$. La base du tronc est munie de contreforts épais. La cime est assez importante et composée de branches dressées avec un feuillage formant un dôme plus ou moins régulier, très caractéristique, en forme de parasol. Les feuilles sont simples. L'essence se comporte comme une espèce tolérante à l'ombre au stade semis mais devient nettement héliophile au stade jeune tige [35].

Recordoxylon speciosum (Caesalpiniaceae) est une essence d'ombre au stade jeune et de lumière au stade adulte. Elle préfère les terrains sains de la forêt primaire. Cette essence possède une régé- 
nération abondante grâce à la dissémination de ses graines ailées. Elle possède un fût sub-cylindrique plus ou moins rectiligne de $25 \mathrm{~m}$ de haut muni d'un houppier important, irrégulier. Les feuilles sont isolées, composées et imparipennées.

Sterculia excelsa (Sterculiaceae) est une essence autochore dont le statut dans la succession est mal défini, probablement à cause de l'existence et de la confusion de plusieurs espèces proches. Sans être une pionnière, c'est tout de même une espèce de lumière. Elle se trouve à l'état disséminé en forêts primaires et secondaires. Sa germination est fréquente mais peu d'individus arrivent à l'état adulte. L'essence mesure entre 30 et $35 \mathrm{~m}$ de haut pour un diamètre pouvant atteindre $100 \mathrm{~cm}$; elle possède une cime à feuillage dense. Des feuilles de formes et de dimensions différentes peuvent se rencontrer sur un même rameau.

Virola surinamensis (Myristicaceae) est une essence qu'on trouve sur les terrains marécageux où elle est dominante mais elle se contente parfois de terrains humides. C'est une essence de lumière mais elle n'est pas considérée comme une espèce pionnière. Elle est très fréquente en forêt et mesure $40 \mathrm{~m}$ de haut et 60 à 70 $\mathrm{cm}$ de diamètre avec une cime peu importante. Les feuilles sont simples, alternes, distiques, oblongues.

Vouacapoua americana (Caesalpiniaceae) est une essence de demi lumière. On la rencontre sous forme de poches sur des terrains sains de forêt primaire : crêtes et pentes de collines mais aussi dans des bas-fonds humides (quelques arbres). Les arbres peuvent atteindre 30 à $35 \mathrm{~m}$ de haut. Ils ont des feuilles alternes, composées et imparipennées.

\section{REMERCIEMENTS}

Les auteurs sont très reconnaissants au Cirad-Forêt (Centre de Coopération Internationale en Recherche Agronomique pour le Développement) pour avoir permis l'installation du dispositif expérimental sur sa concession sise à Paracou. Les auteurs remercient tous ceux dont l'aide a permis l'éclosion de ce travail en particulier les membres de l'équipe technique de la station de Recherches Forestières Inra de Guyane : Dufort S., Duchant M.D., Patient A., Louisanna E. et Guinchard L.

\section{RÉFÉRENCES}

[1] Augspurger C.K., Light requirements of neotropical tree seedlings: a comparative study of growth and survival, J. Ecol. 72 (1984) 777--795.

[2] Baker F.S., A revised tolerance table, J. For. 47 (1949) 179-181.

[3] Barigah T.S., Étude du bilan carboné de deux clones de peuplier à croissance contrastée, thèse, Université Paris-Sud, Orsay, 1991.

[4] Barigah T.S., Saugier B., Mousseau M., Guittet J., Ceulemans R., Photosynthesis, leaf area and productivity of 5 popular clones during their establishment year, Ann. Sci. For. 51 (1994) 613-625.

[5] Bariteau M., La régénération naturelle avant et après exploitation sur le dispositif d'expérimentation sylvicole de Paracou en Guyane franÿaise, thèse, université Paris VI, Paris, 1993.

[6] Bazzaz F.A., Carlson R.W., Photosynthetic acclimation to variability in the light environment of early and late successional plants, Oecologia (Berl.) 54 (1982) 313-316.

[7] Bazzaz F.A., Pickett S.T.A., Physiological ecology of tropical succession: a comparative review, Annu. Rev. Ecol. Syst., 11 (1980) 287-310.

[8] Béna P., Essences forestières de Guyane, Bureau agricole et forestier guyanais, 1960

[9] Béreau M., Louisanna E. Garbaye J., 1997 Effect of endomycorrhizas and nematodes on the growth of seedlings of Dicorynia guianensis Amshoff, a tree of the tropical rain forest in French Guiana, Ann. Sci. For., 54 (1960) 271-277.

[10] Boyé M., Cabaussel G., Perrot Y., Climatologie, in : Atlas des Départements Français d'Outre-Mer : 4, La Guyane, CNRS et Orstom, Paris, 36 Planches, 1979. 
[11] Brokaw N., Gap phase regeneration in a neotropical forest, Ph.D. thesis, University of Chicago, USA, 1980.

[12] Burger D.W., Hartin J.S., Hodel D.R., Lukaszewski T.A., Tjosvold S.A., Wagner S.A., Water use in California's ornamental nurseries, Calif. Agric. 41, 1987, 7-8.

[13] Ceulemans R., Impens I., Study of $\mathrm{CO}_{2}$ exchange processes, resistance to carbon dioxide and chlorophyll content during leaf ontogenesis in poplar, Biol. Plant. (Prague) 21 (4) (1979) 302-306.

[14] Ceulemans R. Saugier B., Photosynthesis, in: Raghavendra A.S. (Ed.), Physiology of Trees, John Wiley \& Sons, New York, USA, 1991, pp. 21-50.

[15] Clark D.B., The role of disturbance in the regeneration of neotropical moist forests, in: Bana K.S., Hadley M. (Eds.), Reproductive ecology of tropical forest plants, UNESCO MAB, 1990, pp. 291-315.

[16] Centre Technique Forestier Tropical, Bois des DOM-TOM, Guyane, tome I, Paris, 1989.

[17] Ducrey M., Influence of shade on photosynthetic gas exchange of 7 tropical rain-forest species from Guadeloupe (French West Indies), Ann. Sci. For. 51 (1994) 77-94.

[18] Epron D., Godard D., Cornic G., Genty B., Limitation of net $\mathrm{CO}_{2}$ assimilation rate by internal resistances to $\mathrm{CO}_{2}$ transfer in the leaves of two tree species (Fagus sylvatica L, and Castanea sativa Mill,), Plant Cell Environ. 18 (1995) 43-5l.

[19] Favrichon V., Classification des espèces arborées en groupes fonctionnels en vue de la réalisation d'un modèle de dynamique de peuplement en forêt guyanaise, Rev. Ecol. (Terre Vie), 49 (1994) 379-403

[20] Field C., Leaf age effects on stomatal conductance, in: Zeiger E., Farquhar G.D., Cowan I.R. (Eds.), Stomatal Function, Stanford University Press, Stanford, California, 1987, pp. 367-384.

[21] Givnish T.J., Adaptation to sun and shade : a whole plant perspective, Aust. J. Plant Physiol. 15 (1988) 63-92.

[22] Granier A., Huc R., Barigah T.S., Transpiration of natural rainforest and its dependence on climatic factors, Agric. For. Meteorol. 78 (1996) 19-29.

[23] Guehl J,-M. Garbaye J., The effects of ectomycorrhizal status on carbon dioxide assimilation capacity, water-use efficiency and response to transplanting in seedlings of Pseudotsuga menziesii (Mirb) Franco, Ann. Sci. For. 21 (1990) 55I-563.

[24] Hett J.M., Loucks O.L., Sugar maple (Acer saccharum Marsh,) seedling mortality, J. Ecol. 59 (1978) 507-520.

[25] Hsiao T.C., Plant response to water stress, Ann. Rev. Plant Physiol. 24 (1973) 519-570.

[26] Huc R., Guehl J.-M., Environmental control of $\mathrm{CO}_{2}$ assimilation rate and leaf conductance in two species of tropical rain forest of French Guiana
(Jacaranda copaia D, don and Eperua falcata Aubl.), Ann. Sci. For. 46 (1989) 443s-447s.

[27] Huc R., Ferhi A., Guehl J.-M., Pioneer and late stage tropical rainforest tree species (French Guiana) growing under common conditions differ in leaf gas exchange regulation, carbon isotope discrimination and leaf water potential, Oecologia (Berl.) 99 (1994) 297-305.

[28] Isebrands J.G., Nelson N.D., Crown architecture of short rotation intensively cultured Populus, II, Branch morphology and distribution of leaves and specific leaf weight within the crown of Populus "Tristis" as related to biomass production, Can. J. For. Res. 12 (1982) 853-864.

[29] Jarvis P.G., Edwards W.R., Talbot H., Models of plant and crop water use, in : Rose D.A., Charles-Edwards D.A. (Eds.), Mathematics and Plant Physiology, Academic Press, London, 1981, 151-194.

[30] Knight D.H., A phytosociological analysis of species-rich tropical forest on Barro Colorado Island, Panama, Ecological Monographs, 45 (1975) 259-284.

[31] Langenheim J.H., Osmond C.B., Brooks A., Ferrar P.J., Photosynthetic responses to light in seedlings of selected Amazonian and Australian rainforest tree species, Oecologia (Berl.) 63 (1984) 215-224.

[32] McMillen G.G., McClendon J.H., Dependence of photosynthetic rates on leaf thickness in deciduous woody plants grown in sun and shade, Plant Physiol. 72 (1983) 674-678.

[33] Médina E., Mooney H.A., Vasquez-Yanes C., in: Junk W. (Ed.), Physiological Ecology of Plants of the Wet Tropics, The Hague, the Netherlands, 1984.

[34] Michael D.A., Dickmann D.I., Gottschalk K.W., Nelson N.D., Isebrands J.G., Determining photosynthesis of tree leaves in the field using a portable ${ }^{14} \mathrm{CO}_{2}$ apparatus: Procedures and Problems, Photosynthetica, 19 (1985) 98-108.

[35] Montpied P., La régénération naturelle en forêt tropicale humide : effet de traitements sylvicoles d'intensité variable, in : Recherche sur les écosystèmes forestiers de guyane, Actes du séminaire interne de Silvolab avril 1994, édition provisoire, 1995 .

[36] Mooney H.A., The carbon balance of plants, Annu. Rev. Ecol. Syst. 3 (1972) 315-346.

[37] Nicholson D.I., Light requirements of seedlings of five species of Dipterocarpaceae, Malayan Forester 23 (1960) 344-356.

[38] Nobel P.S., Leaf anatomy and water use efficiency, in: Turner N.C., Kramer P.J. (Eds.), Adaptation of Plants to Water and High Temperature stress, John Wiley and sons, New York, 1980, 43-52.

[39] Okafo O.A., Hanover J.W., Comparative photosynthesis and respiration of trembling and big- 
tooth aspens in relation to growth and development, For. Sci. 24 (1978) 103-109.

[40] Oldeman R.A.A., Van Dijk J., Diagnosis of the temperament of tropical rain forest trees, in : Gomez-Pompa A., Whitmore T.C., Hadley M. (Eds.), Rain Forest Regeneration and Management, MAB UNESCO Parthenon Publishing Group, Camforth, UK, 1991, pp. 21-65.

[41] Pearcy R.W., Photosynthetic gas exchange responses of Australian tropical forest trees in canopy, gap and understorey microenvironments, Funct. Ecol. I (1987) 169-178.

[42] Ramos J., Grace J., The effects of shade on the gas exchange for four tropical trees from Mexico, Funct. Ecol. 4 (1990) 667-677.

[43] Reighard G.L., Hanover J.W., Shoot and root development and dry matter partitioning in Populus grandidentata, $P$. tremulödes and P. smithii, Can. J. For. Res. 20 (1990) 849-852.

[44] Riddoch I., Grace J., Fasehun E., Riddoch B., Lapido D.O., Photosynthesis and successional status of seedlings in a tropical semi-decidious rain forest in Nigeria, J. Ecol. 79 (1991) 491-503.

[45] Salager J.L., Salager D., Blanc P., Roy J., Capacité photosynthétique d'arbres dans une canopée tropicale : comparaison avec des espèces pionnières, in : Hallé F., Blanc P. (éd.), Biologie d'une canopée de forêt tropicale, 1990, pp. 150-157.

[46] Sasaki S., Mori T., Growth responses of Dipterocarp seedlings to light, Malay. For. 44 (1981) 319-345.
[47] Smith H., Sensing the light environment: the functions of the phytochrome family, in: Kendrick R.E., Kronenberg G.H.M. (Eds), Photomorphogenesis in Plants, Kluwer Academic Publishers, Netherlands, 2nd edition, 1994, pp. $377-416$.

[48] Strauss-Debenedetti S., Bazzaz F.A., Photosynthetic characteristics of tropical trees along successional gradients, in: Mulkey S.S., Chazdon R.L., Smith A.P. (Eds.), Tropical Forest Plant Ecophysiology, Chapman \& Hall, International Thompson Publishing Company, London, UK, 1996, pp. 162-186.

[49] Terry N., Waldron L.J., Taylor S.E., Environmental influences on leaf expansion, in: Dale J.E., Milthorpe F.L. (Eds.), The Growth and Functioning of Leaves, Cambridge University Press, Cambridge, UK, 1981, pp. 179-205.

[50] Thompson W.A., Stocker G.C., Kreidman P.E., Growth and photosynthetic response to light and nutrients of Flindersia brayleyana F Muell, a rainforest tree with broad tolerance of sun and shade, Aust. J. Plant Physiol. 15 (1988) 299-375.

[51] Whitmore T.C., Tropical Rain Forests of the Far East, Oxford University Press, Oxford, 1984.

[52] Whitmore T.C., Canopy gaps and the two major groups of forest trees, Ecology, 70 (1989) $356-538$

[53] Zélitch I., The close relationship between net photosynthesis and crop yield, Bioscience 32 (1982) 796-802. 